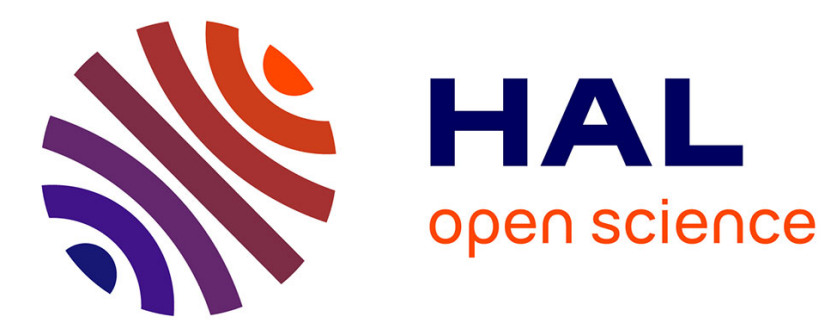

\title{
Speed Advisory and Signal Offsets Control for Arterial Bandwidth Maximization and Energy Consumption Reduction
}

\author{
Giovanni de Nunzio, Gabriel Gomes, Carlos Canudas de Wit, Roberto \\ Horowitz, Philippe Moulin
}

\section{To cite this version:}

Giovanni de Nunzio, Gabriel Gomes, Carlos Canudas de Wit, Roberto Horowitz, Philippe Moulin. Speed Advisory and Signal Offsets Control for Arterial Bandwidth Maximization and Energy Consumption Reduction. IEEE Transactions on Control Systems Technology, 2017, 25 (3), pp.875-887. 10.1109/TCST.2016.2577002 . hal-01332172

\section{HAL Id: hal-01332172 https://hal.inria.fr/hal-01332172}

Submitted on 15 Jun 2016

HAL is a multi-disciplinary open access archive for the deposit and dissemination of scientific research documents, whether they are published or not. The documents may come from teaching and research institutions in France or abroad, or from public or private research centers.
L'archive ouverte pluridisciplinaire HAL, est destinée au dépôt et à la diffusion de documents scientifiques de niveau recherche, publiés ou non, émanant des établissements d'enseignement et de recherche français ou étrangers, des laboratoires publics ou privés. 


\title{
Speed Advisory and Signal Offsets Control for Arterial Bandwidth Maximization and Energy Consumption Reduction
}

\author{
Giovanni De Nunzio, Gabriel Gomes, Carlos Canudas de Wit, Roberto Horowitz, Philippe Moulin
}

\begin{abstract}
The problem of maximizing bandwidth along an arterial is here addressed by use of two combined control actions: traffic light offsets and recommended speeds. The optimization problem has been enriched in order to account for traffic energy consumption and network travel time, thus avoiding impractical or undesirable solutions. A traffic microscopic simulator has been used to assess the performance of the proposed technique in terms of energy consumption, travel time, idling time, and number of stops. The correlation of theoretical bandwidth with known traffic performance metrics is studied, and an analysis of the Pareto optimum has been carried out to help the designer choose a trade-off in the multi-objective optimization. Finally, an evaluation of the traffic performance at different levels of traffic demand aims at showing the best operation conditions of the proposed strategy. A demand-dependent optimization is proposed.
\end{abstract}

Index Terms-bandwidth maximization, energy consumption, speed advisory, signal coordination, linear programming, traffic performance

\section{INTRODUCTION}

Benefits of traffic light coordination on traffic relief are undeniable. Well coordinated traffic lights can reduce travel times, delays, and unnecessary stops. As a consequence, they improve mobility and access, reduce driver frustration, and energy and fuel consumption. Beneficial effects may be observed also in reduced rerouting through nearby neighborhoods, fewer accidents, improved emergency service, and pollution. In [1], experiments showed that the concentration of fine particles in the air at traffic lights during stops is 29-times higher as compared to free-flow conditions. Also, though the delay time at intersections represents only a minor portion of the entire commuting time, it may contribute up to about $25 \%$ of the total trip emissions.

Many traffic-signals optimization algorithms, both fixedtime based (e.g. TRANSYT) and traffic-responsive (e.g. SCOOT, SCATS), have found wide practical use. The objective of these traffic-management strategies is to reduce the total length of the queues in the monitored network area, and consequently reduce delays. Though delay reduction and minimization of stops are considered as important performance targets, maximization of progression bands (i.e. the green interval that allows uninterrupted traffic flow along the entire

G. De Nunzio, Department of Control Signal and Systems at IFPEN, RueilMalmaison, France, giovanni.de-nunzio@ifpen.fr

G. Gomes, Research Engineer, California PATH, UC Berkeley, USA, gomes@path.berkeley.edu

C. Canudas de Wit, Director of Research at CNRS, GIPSA-Lab, NeCS team, Grenoble, France, carlos.canudas-de-wit@gipsa-lab.fr

R. Horowitz, Professor at Department of Mechanical Engineering, Director of California PATH, UC Berkeley, USA, horowitz@berkeley.edu

P. Moulin, Department of Control Signal and Systems at IFPEN, RueilMalmaison, France, philippe.moulin@ifpen.fr arterial [2]) is still considered essential by many practitioners. For this reason, some later versions of the cited algorithms (e.g. TRANSYT-7F) also incorporate progression measures.

The size of the progression band enabled by a sequence of signalized intersections is known as bandwidth. Maximizing the bandwidth of a signalized arterial corresponds to maximizing the time during which vehicles can drive the entire length of the arterial without stopping. Bandwidth-based signal timings are generally preferred because they meet driver expectations about traffic fluidity [3].

The first mathematical formulation of the bandwidth maximization problem was given by Morgan and Little in [4]. The nature of the problem was combinatorial, and it addressed a simplified two-stage version, thus neglecting more complex phases. An extension of this first work was published by Little in [5], where the problem was formulated for the first time as a mixed-integer linear program (MILP).

This pioneering work evolved into the MAXBAND software [6], which offered as main features the possibility to choose cycle times and select offsets to maximize bandwidth in the two directions of travel, as well as the possibility to deal with triangular networks (i.e. a three-artery triangular loop) and more complex turning phases at the intersections. MAXBAND gave rise to several research efforts and extensions, first in order to solve triangular networks (MAXBAND-86) [7], and then to allow different bandwidths for each segment of a single arterial (MULTIBAND) [8] or a network of arterials (MULTIBAND-96) [9], [10]. Recent research has combined link-based and arterial-based techniques [11], as well as introduced network partitioning algorithms in order to deal with larger networks [3]. The assumption of under-saturated traffic (i.e. all queues at the traffic lights are dissipated within the cycle time) has been relaxed in [12], [13].

All of the mentioned works and software packages for bandwidth maximization are based on the original MILP formulation of [5]. However results provided in [14] show that the arterial bandwidth maximization problem can also be cast as a linear program (LP) without the need for integer variables. This result is based on the observation that the integer unknowns are closely related to the inter-signal travel times, and can therefore be computed a priori if the travel times are known.

In the original formulation of the arterial bandwidth maximization by Little [5] and the MAXBAND software package [6], the speed in the segments of the two-way arterial was allowed to vary as an additional decision variable of the MILP to further improve the progression bands. However both in the software implementation and testing of MAXBAND, the potential benefits induced by the variable design speeds on actual traffic performance were never assessed. Only re- 
cently microscopic traffic simulators are used to assess the benefits of bandwidth maximization on traffic performance. In [2] the maximization of bandwidth is achieved with both lights and speeds control and the performance is evaluated in terms of delay and travel time for small arterials with three intersections. The impact of the recommended speeds on the energy consumption and other performance metrics was not considered.

A general and often misleading assumption made by drivers is that an increase in speed will lead to a proportionate decrease in travel time. However, travel time is more dependent on congestion and roadway design and geometry factors than on the recommended speeds. [15] has pointed out that at traffic density levels where a flow can be maintained, it is theoretically possible that lower recommended speeds could actually bring about a reduction in overall travel time. Reduced speeds are likely to have their greatest safety impact at low to medium levels of traffic density where traffic is periodically able to travel at or near the speed limit. We will also show in this paper that a lower average recommended speed may translate into a similar or lower average travel time, while drastically reducing the environmental impact.

In this work, the two-way arterial bandwidth maximization problem is addressed with a particular focus on the benefits induced by the speed advisory, and on reducing energy consumption. The present mathematical formulation, inspired by the idea in [14], [16], allows to solve the one-way bandwidth maximization problem as an LP, even with segment speeds as decision variables. However, as we will see, the two-way problem with internal offsets constraints presents difficulties that make necessary the formulation of the problem as an MILP. The first contribution of the paper lies in the addition of terms representing traffic energy consumption and network travel time to the objective function of the two-way arterial bandwidth maximization. The segment speeds, as additional control action, allow to reach higher theoretical bandwidths but might induce driving discomfort and higher energy consumption if the variability of the recommended speeds is too high. Furthermore, optimal solutions with low speeds and high travel time are to be avoided, in trade-off with the energy consumption. The second contribution of the paper is given by the extensive evaluation of the benefits of bandwidth maximization via a microscopic traffic simulator. Bandwidth is a theoretical quantity and a correlation with known traffic performance metrics needs to be established in order to justify its use. The combined control of offsets and speed advisory is shown to have a large impact on energy consumption without affecting the travel time. Lastly, an analysis of the traffic performance at different levels of traffic demands has been conducted, testing both under-saturated traffic conditions with the existence of a green wave, and saturated conditions. The goal of this analysis is to identify the best operation conditions of the presented approach, assess the performance degradation with traffic load, and, most importantly, propose a demanddependent optimization. Several strategies were compared to the presented one in order to assess its performance.

The paper is organized as follows. Section II describes the problem and the notation. Section III gives the mathematical

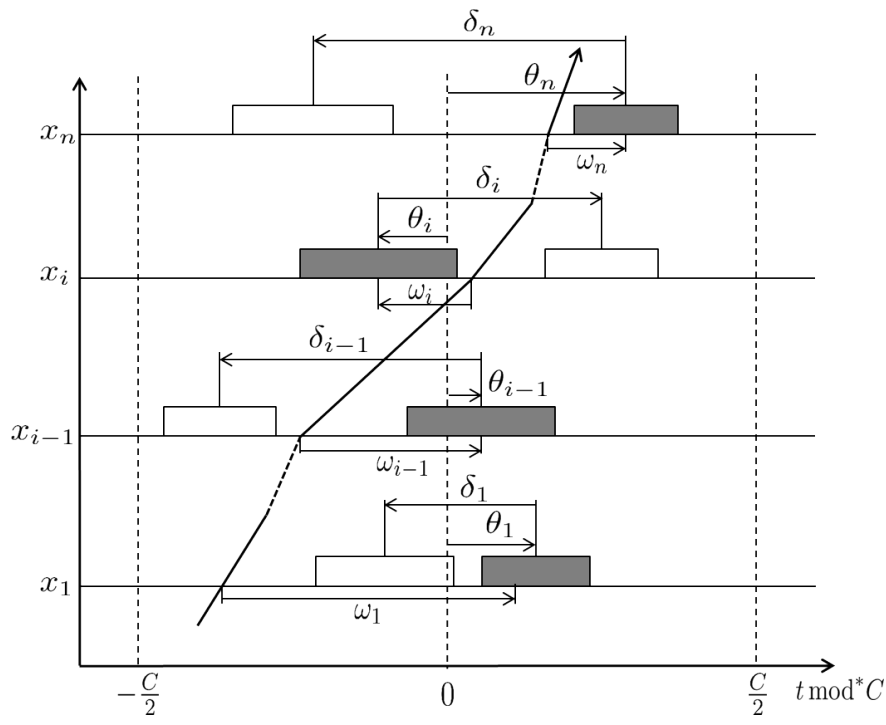

Figure 1: Problem notation. Green windows of the outbound direction of travel are shaded. Green windows in the inbound direction are in white. The travel speed trajectory and the basic notation is indicated only for the outbound direction for clarity.

formulation of the optimization problem. The simulation setup is presented in Section IV. Experiments and results are shown in Section V. Conclusions are given in Section VI.

\section{Problem Setup}

A two-way arterial with $n$ signalized intersections is considered. The two opposing directions of travel are referred to as inbound and outbound. Intersections are located at $x_{1}<x_{2}<\ldots<x_{n}$, with the subscript index increasing in the outbound direction. All quantities related to the inbound direction are denoted with an overbar. The travel speeds in the $n-1$ segments are assumed to be equal to the recommended speeds, and denoted as $v_{i}$ and $\bar{v}_{i}$. This assumption allows for a deterministic and clearer problem formulation, but it will be naturally relaxed in the simulation study, where the vehicles undergo transients to react to perturbations and to converge to the recommended speeds.

The outbound and inbound travel times on segment $i$ are defined as:

$$
\begin{aligned}
& t_{i}=\frac{L_{i}}{v_{i}}>0, \quad i \in\{1, \ldots, n-1\} \\
& \bar{t}_{i}=-\frac{L_{i}}{\bar{v}_{i}}<0, \quad i \in\{1, \ldots, n-1\}
\end{aligned}
$$

with the segment length being $L_{i}=x_{i+1}-x_{i}$.

The $n$ signals are to be coordinated with a common cycle time $C$, which is assumed throughout this paper to be given and fixed. All signals are also on a fixed schedule and they release vehicles in pulses that travel without diffusing. Denote with $g_{i}$ and $\bar{g}_{i}$ the green times at intersection $i$ in the outbound and inbound direction respectively.

The mapping of time from the real domain onto the interval $[-C / 2, C / 2]$ is performed with a non-standard modulo operator which will be denoted $\bmod ^{*}$ (see [14] for details). The absolute offsets $\theta_{i}\left(\bar{\theta}_{i}\right)$ are defined as the displacement 
of the center of the green window of the outbound (inbound) direction at intersection $i$ with respect to a fixed coordinate system (see Fig. 1). The absolute offsets are in $[-C / 2, C / 2]$. The relative displacement of the centers of the inbound and outbound green windows is defined as,

$$
\delta_{i}=\left(\bar{\theta}_{i}-\theta_{i}\right) \bmod ^{*} C
$$

In addition to the absolute offsets, the relative offsets $\omega_{i}$ and $\bar{\omega}_{i}$ are defined. These are measured with respect to the outbound and inbound moving coordinate frames, which travel at speeds $v_{i}$ and $\bar{v}_{i}$ respectively. The moving coordinate frame is a reference frame ideally attached to the stream of vehicles moving in each direction of travel. At the level of abstraction desired for the problem formulation, note that the moving frame does not stop at the red signals. Therefore, the outbound relative offset $\omega_{i}$ is defined as the time between the passage of the outbound moving coordinate frame and the center of the nearest outbound green window. A corresponding definition applies to the inbound relative offset. The relative offsets are also in $[-C / 2, C / 2]$.

The conversion between absolute and relative offsets may be derived as follows:

$$
\left(\omega_{i-1}-\theta_{i-1}+\theta_{i}-\omega_{i}-t_{i-1}\right) \bmod ^{*} C=0
$$

Therefore the following recursive formula for relative offsets applies:

$$
\omega_{i}=\left(\omega_{i-1}-\theta_{i-1}+\theta_{i}-t_{i-1}\right) \bmod ^{*} C
$$

which eventually yields:

$$
\omega_{i}=\left(\omega_{1}-\theta_{1}+\theta_{i}-\sum_{k=1}^{i-1} t_{k}\right) \bmod ^{*} C
$$

The inverse formula allows to determine the absolute offsets from the relative offsets:

$$
\theta_{i}=\left(\theta_{1}-\omega_{1}+\omega_{i}+\sum_{k=1}^{i-1} t_{k}\right) \bmod ^{*} C
$$

These formulas apply with overbars for the inbound direction. Because the map between relative and absolute offsets is invertible, we are free to formulate the bandwidth maximization problem in terms of either set of unknowns.

Bandwidth is a theoretical quantity defined as the size of the time window in which a pulse of vehicles can travel the length of the arterial without stopping.

\section{PROBLEM FORMULATION}

The objective of this work is to solve the two-way bandwidth maximization problem, using both offset and speed control. The degrees of freedom of the optimization will be the relative offsets $\omega_{i}$ and $\bar{\omega}_{i}$, and the travel times $t_{i}$ and $\bar{t}_{i}$.

The two-way problem has been formulated in [14] as a continuous linear program, without use of integer variables, exploiting the a priori information about the speeds in each segment of the arterial network. Only the offsets were used as decision variables. This work extends the previous result by introducing the speeds in the different segments of the network as additional decision variables. It will be shown that a higher bandwidth is achieved thanks to speed advisory control. However, the objective of the current analysis is also to avoid impractical solutions, as well as to evaluate the actual benefits of the maximization of the theoretical bandwidth on traffic performance. In particular, the optimization will account for traffic energy consumption, travel time and driver comfort.

In order to express both $b$ and $\bar{b}$ in terms of the relative offsets, the green windows are translated along the outbound and inbound coordinate frames to $x=x_{1}$. This operation induces a mapping from $\delta_{i}$ to $\delta_{i}^{0}$ with $i \in\{1, \ldots, n\}$. The mapping is given by:

$$
\delta_{i}^{0}=\left(\delta_{i}+\sum_{k=1}^{i-1}\left(t_{k}-\bar{t}_{k}\right)\right) \bmod ^{*} C
$$

with $\delta_{i}^{0} \in[-C / 2, C / 2]$. The equality constraint that fixes the internal offset is given by:

$$
\omega_{i}-\bar{\omega}_{i}=\delta_{1}^{0}-\delta_{i}^{0}
$$

In vector notation:

$$
\bar{\omega}=\omega-\delta
$$

with $\omega \triangleq\left(\omega_{1}, \ldots, \omega_{n}\right)$ and $\delta \triangleq\left(\delta_{1}^{0}, \delta_{1}^{0}-\delta_{2}^{0}, \ldots, \delta_{1}^{0}-\delta_{n}^{0}\right)$.

\section{Directional Interference Constraints}

The progression band, in the case of fixed green times and no platoon dispersion, is equivalent to the intersection of all of the green intervals, measured with respect to the moving coordinate frame. Hence, as was demonstrated in [14], the bandwidth is the size of the intersection of the following set of intervals:

$$
\left\{\left[\omega_{i}-g_{i} / 2, \omega_{i}+g_{i} / 2\right]: i \in\{1, \ldots, n\}\right\}
$$

This leads to a simple expression for the outbound bandwidth:

$$
b\left(\omega_{1}, \ldots, \omega_{n}\right)=\max \left(0, \min _{i, j \in\{1, \ldots, n\}}\left(\omega_{i}-\omega_{j}+g_{i, j}\right)\right)
$$

where $g_{i, j} \triangleq\left(g_{i}+g_{j}\right) / 2$. Equation (11) is the solution to the following linear program:

$$
\begin{array}{ll}
\max & b \\
\text { s.t. } & b \leq g_{i, j}+\omega_{i}-\omega_{j} \\
& b \geq 0
\end{array}
$$

Similarly, the inbound bandwidth can be found with:

$$
\begin{array}{cl}
\max & \bar{b} \\
\text { s.t. } & \bar{b} \leq \bar{g}_{i, j}+\bar{\omega}_{i}-\bar{\omega}_{j} \\
& \bar{b} \geq 0
\end{array}
$$

By combining the two, and imposing the constraint (10), the constraints can be written as:

$$
\begin{array}{ll}
b \leq g_{i, j}+\omega_{i}-\omega_{j}, & \forall i \neq j \in\{1, \ldots, n\} \\
\bar{b} \leq \bar{g}_{i, j}+\omega_{i}-\omega_{j}+\delta_{i}^{0}-\delta_{j}^{0}, & \forall i \neq j \in\{1, \ldots, n\}
\end{array}
$$


and in matrix form:

$$
\begin{aligned}
& b \mathbf{1} \leq \Omega \cdot \omega+\frac{1}{2}|\Omega| \cdot \mathbf{g} \\
& \bar{b} \mathbf{1} \leq \Omega \cdot \omega+\Delta \cdot \delta^{\mathbf{0}}+\frac{1}{2}|\Omega| \cdot \overline{\mathbf{g}}
\end{aligned}
$$

where $\Omega, \Delta \in \mathbb{R}^{n(n-1) \times n}$. The upper and lower bounds for the bandwidths are:

$$
\begin{array}{ll}
b \leq g^{*}, & b \geq 0 \\
\bar{b} \leq \bar{g}^{*}, & \bar{b} \geq 0
\end{array}
$$

where $g^{*}=\min \left(g_{i}\right)$ and $\bar{g}^{*}=\min \left(\bar{g}_{i}\right)$. We have therefore a total of $[2 n(n-1)+4]$ constraints for the bandwidths.

\section{Travel Time Constraints}

The travel times in the two directions $t_{i}$ and $\bar{t}_{i}$ are now decision variables of the optimization problems. The maximum and minimum speed limits are assumed to be the same in both directions, and therefore the bounds of the travel times will depend on the length of the segments. These constraints can be written in vector form as follows:

$$
\begin{aligned}
\mathbf{t}_{\min } & \leq \mathbf{t} \leq \mathbf{t}_{\max } \\
-\mathbf{t}_{\max } & \leq \overline{\mathbf{t}} \leq-\mathbf{t}_{\text {min }}
\end{aligned}
$$

where the vector of travel times is $\mathbf{t}=\left(t_{1}, \ldots, t_{n-1}\right)$, and the bounds on the travel times are:

$$
t_{i, \min }=\frac{x_{i+1}-x_{i}}{v_{\max }}, \quad t_{i, \max }=\frac{x_{i+1}-x_{i}}{v_{\min }}
$$

Analogous equations apply to the inbound direction. This defines $4(n-1)$ constraints.

\section{Internal Offset Constraints}

In this framework, the displaced internal offsets $\delta_{i}^{0}$ are not known a priori, but depend on decision variables $t_{i}$ and $\bar{t}_{i}$ through (8). The difficulty is represented by the modulo operator, which has to be accounted for in the optimization.

Constraints with modulo operator break the standard continuous form of the problem, which now becomes an integer programming problem. Constraint (8) can be rewritten as:

$$
\delta_{i}+\sum_{k=1}^{i-1}\left(t_{k}-\bar{t}_{k}\right)=\alpha_{i} C+\delta_{i}^{0}
$$

where $\alpha_{i} \in \mathbb{N}$, with $i \in\{2, \ldots, n\}$, is a new integer decision variable. Note that the $\delta_{i}$ are given, $\delta_{1}^{0}=\delta_{1}$ and therefore $\alpha_{1}=0$. The constraints will be constructed as follows:

$$
\begin{aligned}
& \delta_{1}^{0}=\delta_{1} \\
& \delta_{2}^{0}=\delta_{2}+t_{1}-\bar{t}_{1}-\alpha_{2} C \\
& \delta_{3}^{0}=\delta_{3}+t_{1}-\bar{t}_{1}+t_{2}-\bar{t}_{2}-\alpha_{3} C
\end{aligned}
$$

In matrix form, bringing the unknowns on the left-hand side, the $n$ constraints on $\delta^{0}$ are as follows:

$$
\delta^{\mathbf{0}}-\Psi \cdot \mathbf{t}+\Psi \cdot \overline{\mathbf{t}}+\alpha \cdot C=\delta
$$

where the matrix $\Psi \in \mathbb{R}^{n \times(n-1)}$, and the vector of integer variables is $\alpha=\left[0, \alpha_{2}, \ldots, \alpha_{n}\right]^{T}$.

\section{Optimization Problem}

As already mentioned, the objective is to maximize bandwidth in the two directions (i.e. sum of the bandwidths). The problem will be solved with respect to the outbound relative offsets and the transformed internal offsets (i.e. offset control), the travel times (i.e. speed advisory), and the integer variables that keep count of the number of cycles. Therefore the number of unknowns of the optimization problem is $5 n-1$ :

$$
\begin{aligned}
\mathbf{x}= & {\left[b, \bar{b}, \omega_{1}, \ldots, \omega_{n}, \delta_{1}^{0}, \ldots, \delta_{n}^{0}, t_{1}, \ldots, t_{n-1},\right.} \\
& \left.\bar{t}_{1}, \ldots, \bar{t}_{n-1}, \alpha_{2}, \ldots, \alpha_{n}\right]^{T}
\end{aligned}
$$

Besides the maximization of the bandwidth, it is also of interest to minimize the variance of the speed advisory, as well as the theoretical travel time. The former may be thought of as a control variance term, or as a comfort term because the drivers would be less willing to follow a highly variable speed advisory. This term also serves to reduce vehicle accelerations that increase energy consumption. The second term drives the optimization away from trivial solutions with impractically low speeds. These terms may be written as a function of $\mathbf{x}$.

Smoothness Term: The speed difference between adjacent segments induced by the speed advisory control can be seen as a proxy for the acceleration at the intersection. Acceleration has major impact on the energy consumption. Therefore, by discouraging large variations in speed between segments, the energy consumption associated with the speed advisory is minimized. The quantity to be minimized for the outbound direction is:

$$
\left\|\frac{L_{i}}{t_{i}}-\frac{L_{i+1}}{t_{i+1}}\right\|_{1}, \quad i \in\{1, \ldots, n-2\}
$$

The same expression applies for the inbound direction, with $\bar{t}_{i}$ and $\bar{t}_{i+1}$. In order to avoid the introduction of nonlinear terms in the objective function, we will approximate (24) with a linear function corresponding to its numerator. Therefore the smoothness term of the objective function can be rewritten as:

$$
\left\|L_{i} t_{i+1}-L_{i+1} t_{i}\right\|_{1}, \quad i \in\{1, \ldots, n-2\}
$$

which can be expressed in matrix form with:

$$
\left\|\mathcal{L}^{*} \cdot \mathbf{t}\right\|_{1}
$$

where $\mathcal{L}^{*} \in \mathbb{R}^{(n-2) \times(n-1)}$ is a matrix of this form:

$$
\mathcal{L}^{*}=\left[\begin{array}{cccccc}
-L_{2} & L_{1} & 0 & \ldots & 0 & 0 \\
\vdots & & & & & \vdots \\
0 & \ldots & -L_{i+1} & L_{i} & 0 & \ldots \\
\vdots & & & & & \vdots \\
0 & 0 & \ldots & 0 & -L_{n-1} & L_{n-2}
\end{array}\right]
$$

Analogously for the inbound direction.

This simplification does not affect the convexity of the problem, and in particular does not modify the minimum of the smoothness term, which will still be reached when the speeds in adjacent road segments are equal. The approximation will change the shape of the function away from the minimum, but its convexity will still drive the optimization towards the desired point. 
Travel Time Term: In order to minimize the theoretical travel time in the network induced by the speed advisory, the $L_{1}$-norm of $t$ is also to be minimized. Therefore the travel time term for the outbound direction in vector form is:

$$
\|\mathbf{t}\|_{1}
$$

Analogously for the inbound direction.

Final Formulation: The problem can be finally formulated as a nonlinear integer program as follows:

$$
\begin{cases}\max _{\mathbf{x}} & b+\bar{b}-\bar{\lambda}_{1}\left(\left\|\mathcal{L}^{*} \mathbf{t}\right\|_{1}+\left\|\mathcal{L}^{*} \overline{\mathbf{t}}\right\|_{1}\right)-\bar{\lambda}_{2}\left(\|\mathbf{t}\|_{1}+\|\overline{\mathbf{t}}\|_{1}\right) \\ \text { s.t. } & b \mathbf{1} \leq \Omega \cdot \omega+\frac{1}{2}|\Omega| \cdot \mathbf{g} \\ & \bar{b} \mathbf{1} \leq \Omega \cdot \omega+\Delta \cdot \delta^{\mathbf{0}}+\frac{1}{2}|\Omega| \cdot \overline{\mathbf{g}} \\ & 0 \leq b \leq g^{*} \\ & 0 \leq \bar{b} \leq \bar{g}^{*} \\ & \mathbf{t}_{\min } \leq \mathbf{t} \leq \mathbf{t}_{\max } \\ & -\mathbf{t}_{\max } \leq \overline{\mathbf{t}} \leq-\mathbf{t}_{\min } \\ & \delta^{\mathbf{0}}-\Psi \cdot \mathbf{t}+\Psi \cdot \overline{\mathbf{t}}+\alpha \cdot C=\delta\end{cases}
$$

The objective function can be linearized by introducing slack variables that behave as maximum bounds for the $L_{1}$ norms [17]. Four such variables $\gamma \in \mathbb{R}^{(n-2)}, \bar{\gamma} \in \mathbb{R}^{(n-2)}$, $\tau \in \mathbb{R}^{(n-1)}$ and $\bar{\tau} \in \mathbb{R}^{(n-1)}$ are introduced such that:

$$
\begin{array}{rlrl}
\left|\mathcal{L}^{*} \cdot \mathbf{t}\right| \leq \gamma & \text { and } & & |\mathbf{t}| \leq \tau \\
\left|\mathcal{L}^{*} \cdot \overline{\mathbf{t}}\right| \leq \bar{\gamma} & \text { and } & |\overline{\mathbf{t}}| \leq \bar{\tau}
\end{array}
$$

Thus, the vector of the unknowns is extended:

$$
\mathbf{z}=[\mathbf{x}, \gamma, \bar{\gamma}, \tau, \bar{\tau}]^{T}
$$

and new constraints appear in the optimization problem.

Therefore the problem can be finally formulated as a mixedinteger linear program with $(9 n-7)$ unknowns and $\left(4 n^{2}+\right.$ $11 n-11)$ constraints:

$$
\left\{\begin{array}{cl}
\max _{\mathbf{z}} & b+\bar{b}-\bar{\lambda}_{1}\left(\mathbf{1}^{T} \gamma+\mathbf{1}^{T} \bar{\gamma}\right)-\bar{\lambda}_{2}\left(\mathbf{1}^{T} \tau+\mathbf{1}^{T} \bar{\tau}\right) \\
\text { s.t. } & b \mathbf{1} \leq \Omega \cdot \omega+\frac{1}{2}|\Omega| \cdot \mathbf{g} \\
& \bar{b} \mathbf{1} \leq \Omega \cdot \omega+\Delta \cdot \delta^{\mathbf{0}}+\frac{1}{2}|\Omega| \cdot \overline{\mathbf{g}} \\
& 0 \leq b \leq g^{*} \\
& 0 \leq \bar{b} \leq \bar{g}^{*} \\
& \mathbf{t}_{\min } \leq \mathbf{t} \leq \mathbf{t}_{\max } \\
& -\mathbf{t}_{\max } \leq \overline{\mathbf{t}} \leq-\mathbf{t}_{\min } \\
& \delta^{\mathbf{0}}-T \cdot \mathbf{t}+T \cdot \overline{\mathbf{t}}+\alpha \cdot C=\delta \\
& \mathcal{L}^{*} \cdot \mathbf{t}-\gamma \leq 0 \\
& -\mathcal{L}^{*} \cdot \mathbf{t}-\gamma \leq 0 \\
& \mathcal{L}^{*} \cdot \overline{\mathbf{t}}-\bar{\gamma} \leq 0 \\
& -\mathcal{L}^{*} \cdot \overline{\mathbf{t}}-\bar{\gamma} \leq 0 \\
& \mathbf{t}-\tau \leq 0 \\
& -\mathbf{t}-\tau \leq 0 \\
& \overline{\mathbf{t}}-\bar{\tau} \leq 0 \\
& -\overline{\mathbf{t}}-\bar{\tau} \leq 0 \\
&
\end{array}\right.
$$

Note that in the current formulation the norms $\left\|\mathcal{L}^{*} \mathbf{t}\right\|$ and $\left\|\mathcal{L}^{*} \overline{\mathbf{t}}\right\|$ are symmetric, thus a reduction in consecutive speeds is penalized as heavily as an increase in speed. The formulation can be generalized by defining an asymmetric norm, so that positive values (i.e. coasting down to a lower speed) are weighted differently from negative values.

This is achieved by introducing a weight $\beta \in[0,1]$ on the constraints containing the slack variables $\gamma$ and $\bar{\gamma}$ in problem (30), as follows:

$$
\begin{aligned}
& \beta \cdot \mathcal{L}^{*} \cdot \mathbf{t}-\gamma \leq 0 \\
& -\mathcal{L}^{*} \cdot \mathbf{t}-\gamma \leq 0 \\
& \mathcal{L}^{*} \cdot \overline{\mathbf{t}}-\bar{\gamma} \leq 0 \\
& -\beta \cdot \mathcal{L}^{*} \cdot \overline{\mathbf{t}}-\bar{\gamma} \leq 0
\end{aligned}
$$

The weight $\beta$ regulates the relative detriment of positive and negative changes in speed. In particular, $\beta<1$ means that decelerations are less penalized than accelerations. In the following, the case with $\beta=1$ is treated.

Furthermore, note that the weighting factors $\bar{\lambda}_{1}$ and $\bar{\lambda}_{2}$ contain the normalization factors in order to have all the terms of objective function in the same order of magnitude. In particular, the weighting factors are defined as follows:

$$
\begin{aligned}
& \bar{\lambda}_{1}=\lambda_{1} \frac{\max \left\{g^{*}, \bar{g}^{*}\right\}}{\lambda_{0}\left(\frac{\max \left\{L_{i}\right\}^{2}}{v_{\min }}-\frac{\min \left\{L_{i}\right\}^{2}}{v_{\max }}\right)} \\
& \bar{\lambda}_{2}=\lambda_{2} \frac{\max \left\{g^{*}, \bar{g}^{*}\right\}}{\lambda_{0} \frac{\max \left\{L_{i}\right\}}{v_{\min }}}
\end{aligned}
$$

where $\max \left\{g^{*}, \bar{g}^{*}\right\}$ is the normalization factor of the bandwidth term, and $\lambda_{0}$ is its weighting factor. In the following, the case with $\lambda_{0}=1$ is treated.

\section{Simulation Setup}

The goal of the simulation campaign is to assess the performance of our optimization and the impact of speed advisory. Specifically, the proposed approach, by means of both offset and speed advisory control, is expected to achieve a higher bandwidth than the offset-based optimization in [14]. Furthermore, the benefits analysis will not be focused only on comparing the levels of total bandwidth achieved. Real traffic performance metrics, such as energy consumption, travel time, idling time at the traffic lights and number of stops, will be analyzed. The objective is to prove that the simple optimization problem hereby formulated will give significant improvements in terms of real traffic performance.

\section{Numerical Implementation}

The two-way bandwidth optimization problem (30) has been implemented in Matlab, using the native solver intlinprog for MILPs. For the sake of comparison, the problem was always solved along with the one formulated in [14] in order to directly observe the differences between the two formulations and the benefits introduced by the speed advisory in terms of theoretical bandwidth. The two optimization problems are designed in such a way that the theoretical bandwidth is always 
at least the size of the shortest green time in the network. In other words, if the result of the optimization yields:

$$
b+\bar{b}<\min \left\{g^{*}, \bar{g}^{*}\right\}
$$

then the direction of travel with the higher bandwidth is set to have a progression band equal to its shortest green time. In this way we ensure that the highest possible theoretical bandwidth is always achieved.

The computation time is negligible: 0.3 seconds for the problem here presented, 0.15 seconds for the problem presented in [14], for an arterial network with 10 intersections. It has also been observed that the MILP here presented scales better with the increasing number of intersections, whereas the simpler offsets-based optimization runs more slowly when $n$ increases. For instance, for $n=20$, the MILP optimization converges in about 0.4 seconds, while the LP converges in about 0.9 seconds. This can be explained by the generally larger space of optimal solutions available when combining offset and speed advisory control. In other words, as $n$ increases, the offset-only optimization search for the solution in a smaller and smaller space, therefore taking longer to converge. On the contrary, the combined optimization converges faster to one possible optimal solution because the search space is larger. Based on this, it is also possible to conclude that the integer variables in the optimization problem under analysis do not affect significantly the convergence time.

The simulations were run with a laptop equipped with an Intel(R) Core(TM) i7-2760QM at $2.40 \mathrm{GHz}$ and $8 \mathrm{~GB}$ of RAM.

\section{Microscopic Traffic Simulator}

Aimsun was adopted as microscopic simulator for the tests. The traffic demand used in the traffic simulation was required to be feasible, in the sense that the green times were sufficient to dissipate the queues in each cycle. A conservative upper bound for the feasible demand is given by:

$$
D \leq D_{\max }=\frac{g^{*}}{C} \varphi_{m}\left(v_{\min }\right) \quad[\mathrm{veh} / \mathrm{h}]
$$

where $g^{*}$ is the minimum of the green times in the signalized corridor, and $\varphi_{m}\left(v_{\min }\right)$ is the demand/supply saturation function for the imposed minimum speed limit [18]. This definition of feasible demand is applied to each direction of travel.

A communication protocol between Aimsun and Matlab was set up in order to retrieve from the microscopic simulator information about the vehicles in the network, at each simulation step. Namely the vehicles send information about their position, current speed and acceleration. These data are used to compute traffic performance metrics. Each one of the performance metrics was obtained per segment and per vehicle completing the trip on the segment. Vehicles that at the end of the simulation time horizon remained in the network, do not fully contribute to the overall performance. After computing the performance per segment and per vehicle, the average performance per segment was obtained. In the following analysis, only the network-wide performance is reported, that is the sum over the two directions of travel of the mean performance in each segment. The performance metrics used in this work are defined as follows:
Travel Time: The time that a vehicle spends in the network. Idling Time: The time during which a vehicle has zero speed.

Number of Stops: The number of idling periods of a vehicle in the network.

Energy Consumption: The energy is defined as the integration over time (i.e. the time the vehicle is in the segment) of the power demand.

$$
E=\int_{0}^{T_{f}} P \mathrm{~d} t=\int_{0}^{T_{f}} b_{1} u v \mathrm{~d} t
$$

The torque $u$ is derived from the longitudinal model of the vehicle [19]:

$$
\begin{aligned}
& \dot{x}=v \\
& \dot{v}=h_{1} u-h_{2} v^{2}-h_{3} v-h_{0}
\end{aligned}
$$

where $u$ is a function of the speed and the acceleration of the vehicle. The constants $b_{i}$ and $h_{i}$ were identified in [20]. Note that such energy consumption model can be also adapted to electric vehicles by adding a term proportional to the square of the torque, representing the electrical losses in the armature of the motor.

\section{EXPERIMENTS}

\section{Bandwidth degradation}

The first experiment aims at showing the degradation of the theoretical bandwidth with the increasing number of signalized intersections on the arterial. Several network parameters have been varied randomly in order to test different sets of green times, segment lengths, and internal offsets. The range of the parameters is shown in Table I.

Table I: Network parameters range for stochastic simulation

\begin{tabular}{lll}
\hline $\mathrm{n}$ & {$[3, \ldots, 15]$} & number of intersections (per direction) \\
$\mathrm{C}$ & 60 & cycle time $[\mathrm{s}]$ \\
$\mathrm{g}$ & {$[0.4,0.6]$} & green signal split ratio \\
$\mathrm{L}$ & {$[225,375]$} & segment lengths $[\mathrm{m}]$ \\
$\delta$ & {$[-C / 2, C / 2]$} & internal offset $[\mathrm{s}]$ \\
{$\left[v_{\min }, v_{\max }\right]$} & {$[15,50]$} & speed limits $[\mathrm{km} / \mathrm{h}]$ \\
\hline
\end{tabular}

In order to extensively explore the space of varying parameters, for each number of intersections $n$, the other parameters (i.e. green split ratio, segment's length, and internal offset) were allowed to vary randomly within the specified ranges. A total of 10,000 simulations per each value of $n$ were run (i.e. 130,000 total different configurations). Furthermore, for each randomly generated configuration, both problem (30) and the two-way bandwidth maximization presented in [14] were solved. The comparison of the theoretical bandwidth achieved by the two optimizations is shown in Fig. 2.

In the graph, the solid lines represent the average bandwidth varying with the number of intersections. The vertical error bars at each value of $n$ represent the standard deviation of the bandwidth calculated over the random set of parameters. As $n$ increases the bandwidth decreases for both types of control 


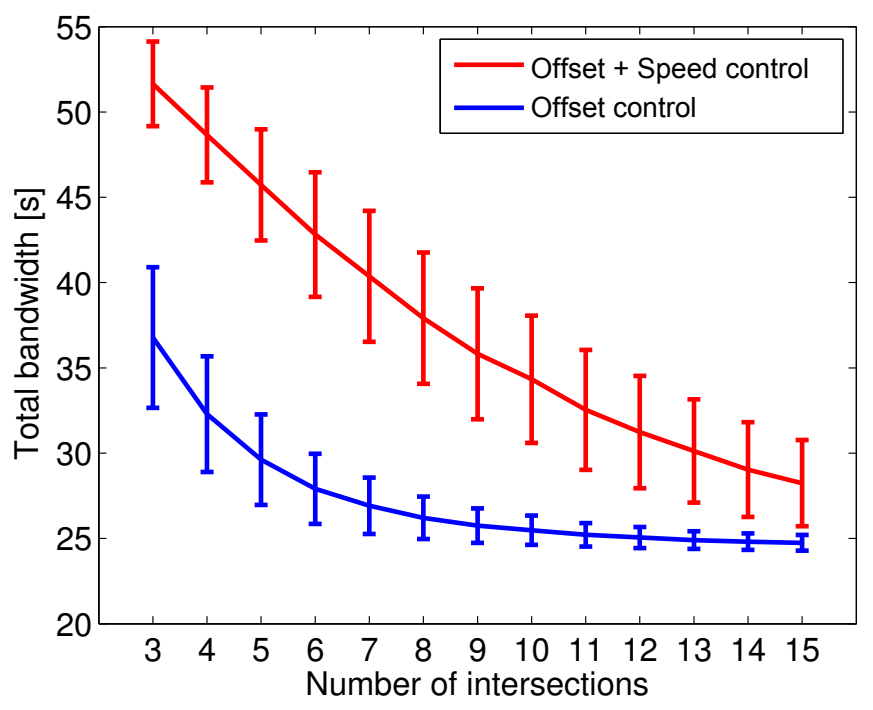

Figure 2: Bandwidth degradation with increasing number of intersections. In blue the offset control strategy in [14].

action. However control of both offsets and recommended speeds results to be significantly more effective, especially for a lower number of intersections. Note that for each $n$, the bandwidth achieved using the proposed approach is always greater or equal to that obtained using only offset control.

The weights used in the optimization are $\lambda_{1}=\lambda_{2}=0.5$. Interestingly, note that the red line would move depending on the selection of weights $\lambda_{1}$ and $\lambda_{2}$. Specifically, when $\lambda_{2}>>0$, the red line would coincide with the blue one, meaning that the presented optimization is forced to select the maximum speeds. When the two weights are set to zero, the red line would move up at its maximum, meaning that the speed advisory would be fully employed by the optimization to maximize only the total bandwidth.

\section{Microscopic simulation}

For the microscopic simulations a single random network configuration was selected. The network parameters utilized in Aimsun are summarized in Table II.

Table II: Network parameters for microscopic simulation

\begin{tabular}{lll}
$n$ & 6 & number of intersections \\
$g$ & {$[33,30,25,28,31,26]$} & outbound green times [s] \\
$\bar{g}$ & {$[33,27,35,27,33,26]$} & inbound green times [s] \\
$L$ & {$[268.1,238.7,311.4,327.5,307]$} & length of the segments [m] \\
$\delta$ & {$[25,-21,-21,-19,-22,-3]$} & internal offsets [s] \\
$D$ & 500 & traffic demand [veh/h] \\
\hline
\end{tabular}

For the choice of macroscopic network parameters (i.e. jam density, congestion speed, etc.), the arterial capacity is $Q=$ $1850 \mathrm{veh} / \mathrm{h}$, and the maximum feasible demand is $D_{\max }=$ $536 \mathrm{veh} / \mathrm{h}$. Therefore, the simulations were conducted using a traffic demand close to the limit of existence of a green wave.

Given these network parameters, the two bandwidth optimization problems were solved numerically, in order to obtain the control parameters (i.e. offsets and speeds) to be tested in the microscopic simulator.

Bandwidth and Traffic Performance: For the assessment of the results of the combined optimization of offsets and recommended speeds, the space of the weights $\lambda_{1}$ and $\lambda_{2}$ of the multi-objective function has been spanned. The results are reported in Fig. 3 and Fig. 4.

In Fig. 3 it is evident how the theoretical bandwidth is monotonically decreasing in the direction of the increasing $\lambda_{1}$ and $\lambda_{2}$. Higher weights on the smoothness term and/or the travel time term in the optimization would achieve smoother and/or higher overall speed profiles between adjacent segments. Therefore, for high values of $\lambda_{2}$ the optimization converges towards the blue area of the bandwidth plot, which is equivalent to the bandwidth achieved by the offset-only optimization, as reported in Table III. The benefits of the speed advisory for bandwidth maximization are evident for lower values of the weights $\lambda_{1}$ and $\lambda_{2}$. In particular, by setting the weights equal to zero, the maximum bandwidth value is achieved, and the full potential of the speed advisory is exploited. The plot of the smoothness term of the objective function (lower values correspond to higher smoothness) shows how it is monotonically decreasing in the direction of increasing $\lambda_{1}$, as expected. On the contrary the travel time term improves (i.e. higher speeds are recommended) monotonically in the direction of increasing $\lambda_{2}$. Note that the abrupt variations in the contour plot of the total bandwidth can be easily explained with the presence of integer variables in the problem.

The traffic performance metrics, computed from the microscopic simulation data, are reported in Fig. 4. It is of interest to observe how the theoretical bandwidth is correlated with actual traffic performance. Energy consumption is low in the area of maximum bandwidth, since higher bandwidth reduces also the idling time and the number of stops. However, energy is optimized for low values of $\lambda_{2}$, showing the positive impact of lower recommended speeds. If $\lambda_{2}$ is increased in order to aim at lower travel time, an increase of $\lambda_{1}$ is required for the energy consumption to stay at its minimum. Therefore, lower and/or smoother recommended speeds are to be preferred for lower energy consumption. Interestingly also travel time is minimized in the area of higher bandwidth, even though the total average recommended speed is lower than the maximum speed limit. This is an interesting demonstration of the fact that higher speeds do not always correspond to lower travel times. For high $\lambda_{1}$ and small $\lambda_{2}$ the travel time increases because the optimization forces the recommended speeds to be as close as possible, without giving priority to high speeds. Idling time and number of stops appear to be the most correlated metrics to bandwidth, and they show overall similar behavior in the $\lambda_{1}$ $\lambda_{2}$ plane in Fig. 3 and Fig. 4. More specifically, the correlation coefficient between bandwidth and idling time is $R_{\mathrm{bi}}=0.95$, and the correlation coefficient between bandwidth and number of stops is $R_{\mathrm{bs}}=0.94$. Also note that where the bandwidth is minimum, all the performance metrics worsen significantly.

When facing a multi-objective optimization it is always challenging to make the right choice of the weighting factors. A Pareto efficiency analysis has been conducted on the 

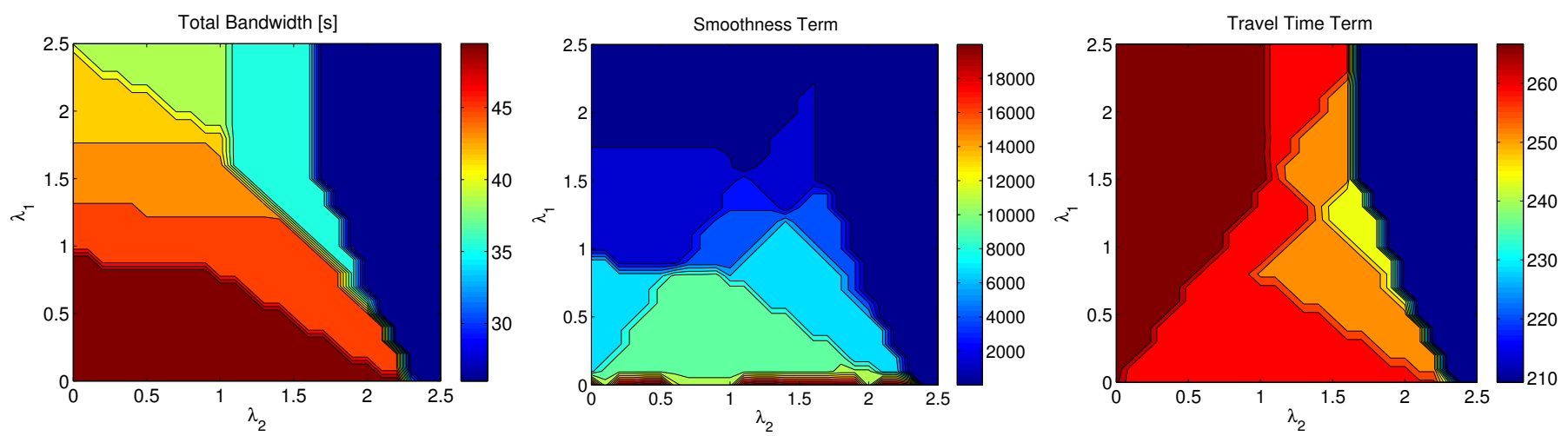

Figure 3: Contour plots of the numerical results. For the theoretical bandwidth, higher values (towards red) are better. For the smoothness term and the travel time term of the objective function, lower values (towards blue) are better.
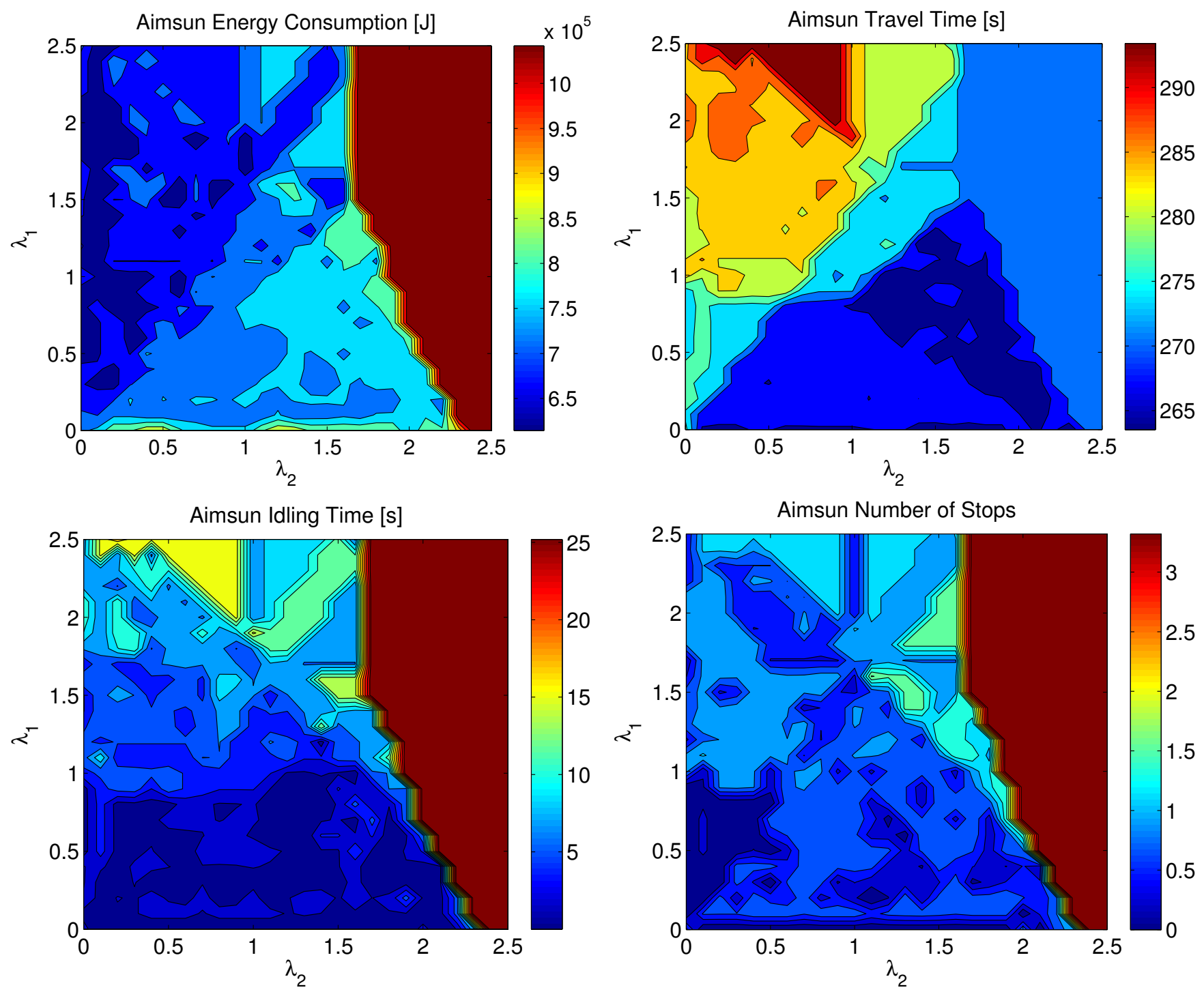

Figure 4: Contour plots of the Aimsun results. The performance metrics reported here (i.e. energy consumption, travel time, idling time, and number of stops) are optimized for lower values (towards blue). 
problem in order to assist the designer in making decisions within the set of points belonging to the Pareto front. The Pareto frontier is defined as the set of points that cannot be said to be strictly worse than any other belonging to the set. All the points on the frontier are equivalently Pareto-efficient. In the case under analysis, the terms of the objective function are three and the Pareto frontier is a surface in a three-dimensional space. In order to find the set of the Pareto-efficient points, a standard non-dominated sorting algorithm was used. The adopted methodology is described as follows. The entire set of solutions obtained from the optimization problem (30) for the three terms of the objective function and for different weights combinations (as shown in Fig. 3) is searched for non-dominated solutions. This gives the Pareto frontier of the optimization problem. The same procedure is run for the set of Aimsun performance metrics. In other words, a Pareto front is found also for the results obtained in Aimsun for energy consumption, travel time, idling time and number of stops. The two Pareto fronts are then mapped onto the $\lambda_{1}-\lambda_{2}$ plane and only the intersection of the two Pareto-efficient sets is considered. The idea is to reduce the set of weight options to the ones that are Pareto efficient both for the theoretical optimization problem and for the actual traffic performance obtained in the microscopic simulator. Within this reduced set, we have chosen the weights that achieve the lowest energy consumption, and obtained $\lambda_{1}=\lambda_{2}=0.4$.

In order to evaluate the performance of the presented optimization, which in the following will be indicated as "Offset+Speed", a comparative study was conducted with preexisting strategies and a reference. The reference used hereafter is a non-optimized case, where the random choice of offsets still respects the internal offset constraint (3). A comparison will be made also with the offset optimization strategy in [14]. Finally, the MAXBAND algorithm will be compared to the proposed strategy. The presented optimization problem can be seen as a generalization of the MAXBAND algorithm in [5][6] with the segment speeds allowed to vary in $\left[v_{\min }, v_{\max }\right]$. In MAXBAND, the formulation for the outbound/inbound arterial problem corresponds to the particular case of $\lambda_{1}=\lambda_{2}=0$ in the presented framework.

Table III: Numerical and Aimsun results comparison

\begin{tabular}{|l|l|l|l|l|l|}
\hline & Numerical & \multicolumn{4}{|c|}{ AIMSUN } \\
\cline { 2 - 7 } & $\begin{array}{l}\text { Theoretical } \\
\text { Bandwidth }\end{array}$ & $\begin{array}{c}\text { Travel } \\
\text { Time }\end{array}$ & $\begin{array}{c}\text { Idling } \\
\text { Time }\end{array}$ & Stops & Energy \\
\hline Non-optimized & N/A & $367.9 \mathrm{~s}$ & $102.8 \mathrm{~s}$ & 6.7 & $1.3 \mathrm{E} 6 \mathrm{~J}$ \\
\hline Offset & $26 \mathrm{~s}$ & $270.3 \mathrm{~s}$ & $26.9 \mathrm{~s}$ & 3.5 & $1.09 E 6 \mathrm{~J}$ \\
\hline MAXBAND & $51 \mathrm{~s}$ & $278 \mathrm{~s}$ & $2.3 \mathrm{~s}$ & 0.7 & $7.48 \mathrm{E} 5 \mathrm{~J}$ \\
\hline Offset+Speed & $51 \mathrm{~s}$ & $268.3 \mathrm{~s}$ & $1.9 \mathrm{~s}$ & 0.8 & $7.03 \mathrm{E} 5 \mathrm{~J}$ \\
\hline
\end{tabular}

The comparative results for both theoretical bandwidth and Aimsun performance metrics are reported in Table III. Besides the significant difference in terms of bandwidth achieved thanks to the introduction of recommended speeds as decision variables, important improvements are achieved also in terms of traffic performance. The presented strategy yields a reduction of the overall network travel time of $1 \%$ with respect to the offset optimization, although the average speed is lower. The idling time and the number of stops at the intersections are almost completely eliminated. The overall energy consumption is reduced by $35.5 \%$. With respect to MAXBAND, the proposed strategy is able to reduce the travel time by $3.5 \%$, and the energy consumption by $6 \%$. Much larger improvement is evidently achieved with respect to the nonoptimized case: travel time is reduced by $27 \%$ and energy consumption is reduced by $46 \%$.

Note that for the considered level of demand (i.e. demand is feasible and equal to $500 \mathrm{veh} / \mathrm{h}$ ) and for the choice of weights in the objective function (i.e. $\lambda_{1}=\lambda_{2}=0.4$ ), the improvement of the presented strategy with respect to MAXBAND is not very large. In the following, the analysis of the performance at different levels of traffic demand will lead to a demand-based optimization and the benefits of the Offset+Speed strategy will further increase.

Table IV: Control variables of the optimization problems

\begin{tabular}{|l|l|l|}
\hline & Offsets [s] & Recommended Speeds $[\mathrm{km} / \mathrm{h}]$ \\
\hline \multirow{2}{*}{ Non-optimized } & $\theta=[-16,-20,-16,-4,-11,25]$ & $v=[50,50,50,50,50]$ \\
& $\bar{\theta}=[9,19,23,-23,27,22]$ & $\bar{v}=[50,50,50,50,50]$ \\
\hline \multirow{2}{*}{ Offset } & $\theta=[0,27,10,-15,25,-17]$ & $v=[50,50,50,50,50]$ \\
& $\bar{\theta}=[25,6,-11,26,3,-20]$ & $\bar{v}=[50,50,50,50,50]$ \\
\hline \multirow{2}{*}{ MAXBAND } & $\theta=[0,-29,18,-12,28,-13]$ & $v=[40,17,35,33,50]$ \\
& $\bar{\theta}=[25,10,-3,29,6,-16]$ & $\bar{v}=[50,50,50,50,50]$ \\
\hline \multirow{2}{*}{ Offset+Speed } & $\theta=[0,21,-4,-24,12,-26]$ & $v=[50,26,29,34,43]$ \\
& $\bar{\theta}=[25,0,-25,17,-10,-29]$ & $\bar{v}=[44,44,50,50,50]$ \\
\hline
\end{tabular}

In Table IV the output of the three optimization problems is reported. The strategy here presented, as well as MAXBAND, shows some variability in the speed advisory, which may cause some drivers to ignore the recommended speed.

Price of Noncompliance with the Speed Advisory: An additional simulation in Aimsun was conducted in order to assess the "price of noncompliance" with the speed advisory. The set of offsets outputted by the optimization for the combined control was implemented, but the vehicles were allowed to drive freely up to the maximum speed limit (i.e. $50 \mathrm{~km} / \mathrm{h}$ ).

Table V: Aimsun traffic performance in the case of noncompliance with the speed advisory

\begin{tabular}{|l|c|c|l|c|}
\hline \multirow{2}{*}{} & \multicolumn{4}{|c|}{ AIMSUN } \\
\cline { 2 - 5 } & $\begin{array}{c}\text { Travel } \\
\text { Time }\end{array}$ & $\begin{array}{c}\text { Idling } \\
\text { Time }\end{array}$ & Stops & Energy \\
\hline Offset+Speed & $260.9 \mathrm{~s}$ & $21.1 \mathrm{~s}$ & 3 & $1.05 \mathrm{E} 6 \mathrm{~J}$ \\
\hline MAXBAND & $274.3 \mathrm{~s}$ & $33.6 \mathrm{~s}$ & 2.7 & $1.07 \mathrm{E} 6 \mathrm{~J}$ \\
\hline
\end{tabular}

From Table V, it is evident how the noncompliance with the speed advisory is very costly in terms of energy consumption, idling time and number of stops, yielding only a slight reduction of the overall travel time.

Variation of Traffic Demand: As previously mentioned, the experiments were conducted at a feasible level of demand, which guarantees the existence of a progression band. However, different levels of traffic demand are very likely to arise in reality and it is important to study the effects on traffic performance. Simulations were run in order to assess the 

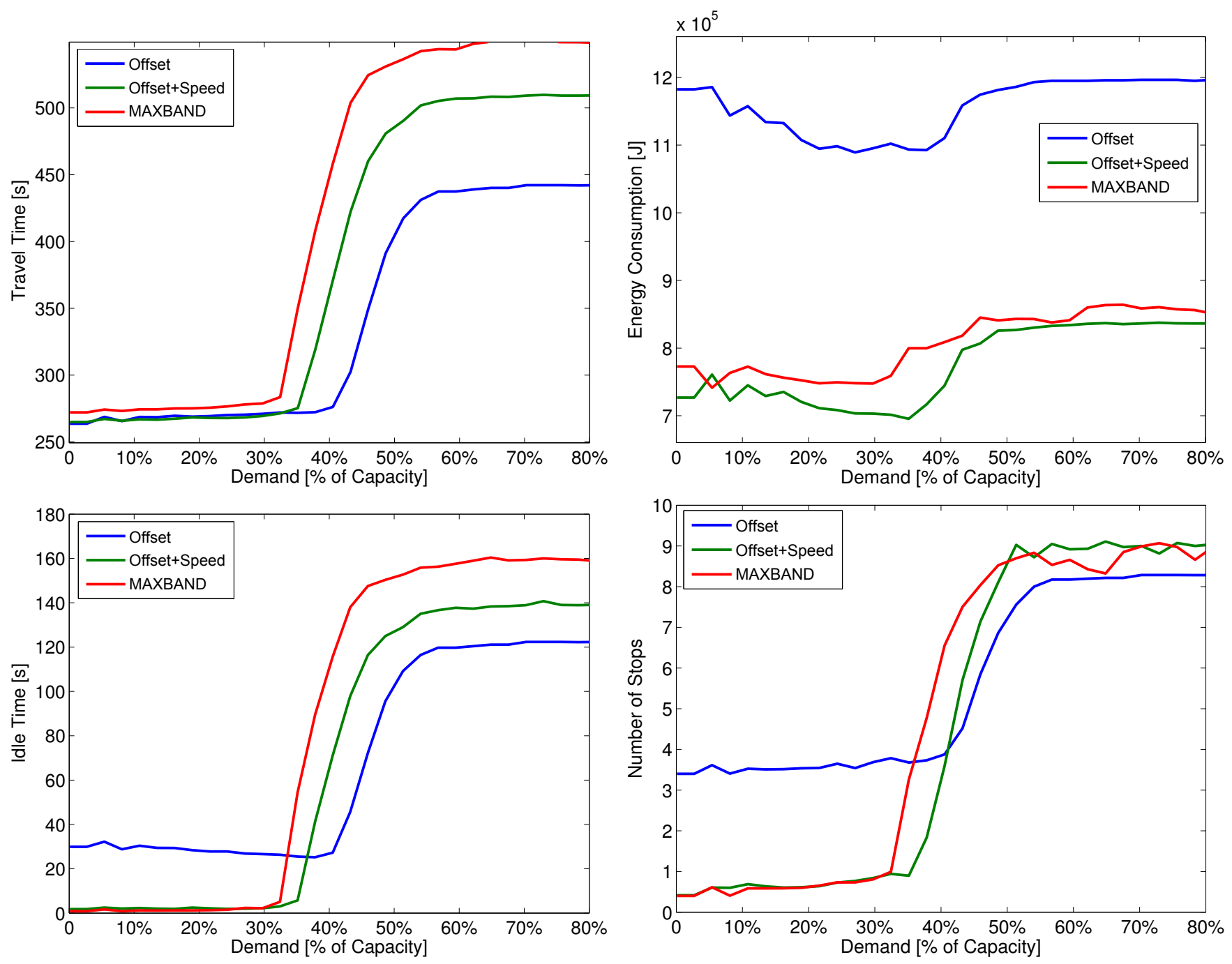

Figure 5: Variation of the traffic performance metrics as a function of the traffic demand.

sensitivity of the traffic performance to a demand up to the limit of feasibility. Infeasible demands were also tested in order to evaluate the impact of heavy traffic on the network optimized by the arterial bandwidth maximization algorithm.

The variation of energy consumption, travel time, idling time, and number of stops, as a function of traffic demand, is reported in Fig. 5. The demand is expressed as a percentage of the arterial capacity. Up to the feasible demand, the queues at each intersection are dissipated within the cycle time, as previously mentioned. For higher demands, queues begin to form at the bottleneck intersection, which corresponds to the traffic light with the smallest outflow. This critical value of demand can be exactly computed, by knowing the traffic light green split ratio and the recommended speed in the segment given by the optimization, as follows:

$$
D_{\text {crit }}=\min \left\{\min \left\{\frac{g_{i}}{C} \varphi_{m}\left(v_{i}\right)\right\}, \min \left\{\frac{\bar{g}_{i}}{C} \varphi_{m}\left(\bar{v}_{i}\right)\right\}\right\}
$$

for $i=1, \ldots, n$. This critical demand corresponds in Fig. 5 to the point at which the performance metrics begin to degrade (i.e. between $30 \%$ and $40 \%$ of the road capacity).
Interestingly, up to the critical demand, the travel time for the strategy here presented is absolutely comparable to the one achieved with offset optimization, even though the speed advisory control induces a lower average speed. This can be explained by looking at the number of stops and the idling time, which are drastically reduced by the speed advisory control. In other words, the presented approach allows to convert the time wasted idling at a red traffic light into a slower approach to the traffic light, without affecting the travel time. The proposed optimization presents also an improvement with respect to the MAXBAND algorithm (i.e. $\lambda_{1}=\lambda_{2}=0$ ), reducing the travel time by $3 \%$ for demands up to the critical one. It should be noted also that the offset-only optimization, with the uncontrolled maximum speed limit, achieves the best performance in terms of travel time for demands beyond the critical one.

As for the energy consumption, the proposed strategy, is consistently better than the offset optimization, yielding an average reduction of about $33 \%$ at any level of demand. An average reduction of about $5 \%$ is also achieved with respect to MAXBAND. It is interesting to note that the average network 

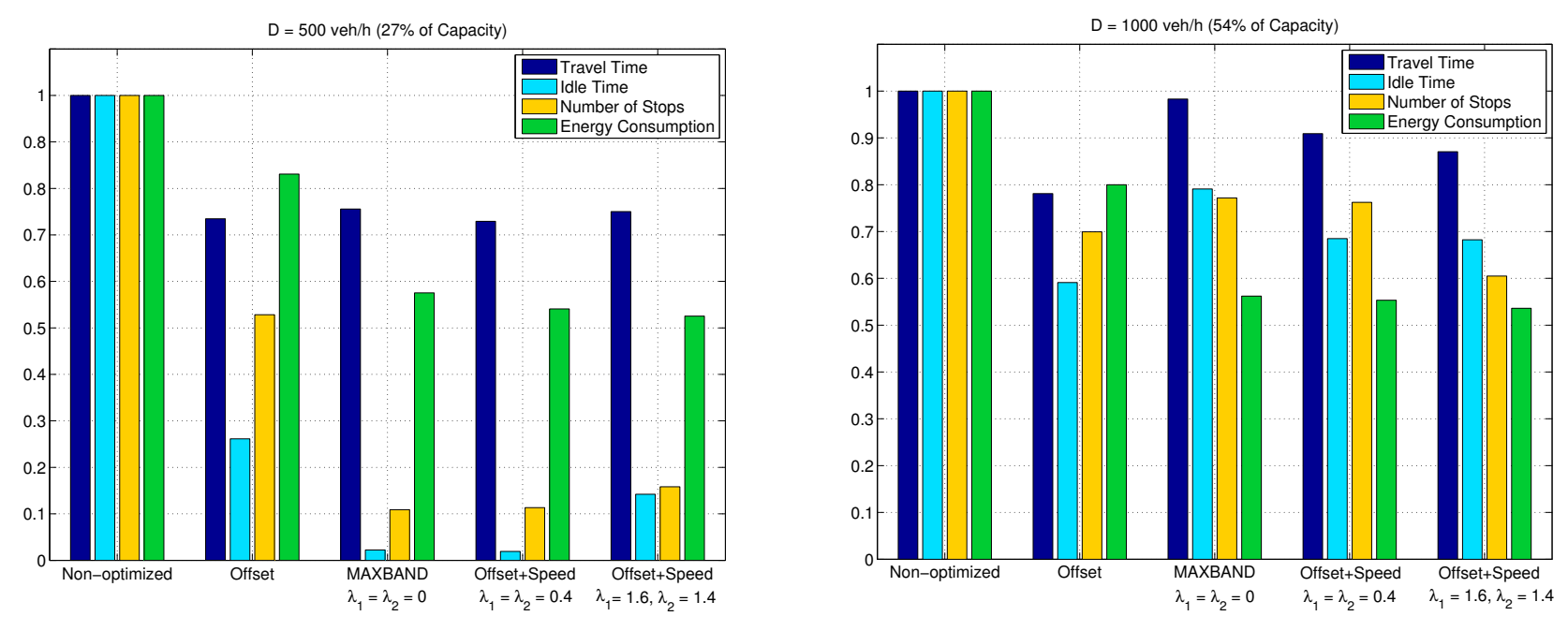

Figure 6: Performance comparison of the demand-based optimization.

energy consumption is lower for demands approaching the critical one. Longer platoons of non-stopping vehicles allow for a better use of the infrastructure and to improve the average traffic performance.

In terms of idling time and number of stops, MAXBAND and the proposed strategy are comparable and allow to almost eliminate stops and idling in the network. However, when the traffic demand grows beyond the critical value, the offset optimization and the higher recommended speeds allow for better performance because the vehicles are able to leave the queues faster.

Therefore, it is possible to conclude that for under-saturated traffic conditions and feasible demands, the proposed optimization of offsets and recommended speeds is able to drastically reduce the traffic energy consumption without affecting the travel time. A more fluid driving experience is also provided by almost completely eliminating stops at red lights.

Demand-Based Optimization: This important result suggests the possibility of a demand-based optimization. An analysis of the Pareto optimum at different levels of traffic demand should indicate different optimal operation conditions and, consequently, different weights $\lambda_{1}$ and $\lambda_{2}$ to be used in the optimization. Experiments were conducted for $D=\{250,500,750,1000\} \mathrm{veh} / \mathrm{h}$, corresponding to $\{14 \%, 27 \%, 41 \%, 54 \%\}$ of road capacity. The performance at each level of demand was studied as in Fig. 4, and the mapping of the Pareto front on the $\lambda_{1}-\lambda_{2}$ plane was obtained. Intuitively, as the traffic conditions become saturated and the original hypothesis of feasible demand does not hold anymore, the Pareto optimum leaves the region of the $\lambda_{1}-\lambda_{2}$ plane corresponding to the maximum theoretical bandwidth. At saturated traffic conditions (as shown in Fig. 5), the travel time is minimized for high-average speeds (i.e. $\lambda_{2}>>0$ and $\lambda_{1}>>0$ ), whereas the energy consumption is minimized for low-average and smooth speeds (i.e. $\lambda_{2} \simeq 0$ and $\lambda_{1}>>0$ ). The demandbased optimization can be summarized as follows. The choice of $\lambda_{1}=\lambda_{2}=0.4$ is Pareto efficient up to saturation, which arises in this scenario at a traffic demand equal to about
$50 \%$ of road capacity. At saturation, therefore for a demand beyond $50 \%$ of road capacity, the previous selection of weights is no longer Pareto efficient. A new set of Pareto-efficient weights that minimize energy consumption was chosen, and we obtained $\lambda_{1}=1.6$ and $\lambda_{2}=1.4$. The recommended speeds yielded by the optimization with such set of weights are: $v=[43,34,34,34,34] \mathrm{km} / \mathrm{h}$ and $\bar{v}=[50,50,50,50,50]$ $\mathrm{km} / \mathrm{h}$.

The traffic performance obtained for the levels of demand $D=500 \mathrm{veh} / \mathrm{h}$ (i.e. under-saturated) and $D=1000 \mathrm{veh} / \mathrm{h}$ (i.e. saturated), normalized with respect to the non-optimized case previously described, is reported in Fig. 6. At under-saturated conditions, as already summarized in Table III, the set of weights $\lambda_{1}=\lambda_{2}=0.4$ is Pareto efficient and outperforms all the competitors. At saturated conditions, the previously used set of weights is no longer Pareto-efficient, and the new set of weights $\lambda_{1}=1.6$ and $\lambda_{2}=1.4$ allows to achieve better performance. In particular, the demand-based optimization improves all four performance metrics with respect to the previous optimization with the original set of weights. Also, the improvement with respect to MAXBAND is larger than in under-saturated conditions: energy consumption is reduced by $5 \%$, the number of stops is reduced by $22 \%$, the idling time is reduced by $14 \%$, and the travel time is reduced by $11 \%$. Evidently, the advantages with respect to the non-optimized case are much larger. The interesting competing strategy in the case of saturated traffic conditions is represented by the offsetonly optimization, due to the trade-off between travel time and energy consumption. As one may notice, the idling time and the number of stops are approximately equivalent in the two strategies, while significant differences are observed in the travel time and the energy consumption. Namely, the demandbased Offset+Speed strategy reduces energy consumption by $33 \%$, while increasing the travel time by $10 \%$. The traffic manager could opt for a different Pareto-efficient combination of weights if willing to reduce the travel time at the expenses of energy consumption. 


\section{CONCLUSIONS}

This work has outlined a solution of the arterial bandwidth maximization by using both offset control and recommended speeds, and by taking into account the effects on energy consumption and network travel time. An extensive evaluation of the proposed solution has been carried out in a microscopic traffic simulator in order to assess the correlation between the theoretical bandwidth and the standard traffic performance metrics. It has been found that higher bandwidth corresponds mainly to lower idling time, number of stops, and energy consumption. The correlation with travel time is weaker and it depends on the applied speed advisory control. The weights used in the objective function allow to find a trade-off between energy consumption and travel time. Finally an analysis of the Pareto front allowed to choose an efficient combination of weights that resulted in drastic energy consumption reduction. It has also been shown that noncompliance with the advised speeds leads to a significant performance degradation. Finally, an analysis of traffic performance at different levels of demand, including infeasible demands, was conducted. It has been shown that the proposed approach outperforms other existing strategies, and in particular, for under-saturated traffic conditions, achieves reduction of energy consumption without affecting the travel time.

Future research will include investigations into more complex networks with cross streets and additional inflows within the arterial, which might open interesting analyses of the benefits of speed advisory on traffic performance.

\section{ACKNOWLEDGMENTS}

The research leading to these results has received funding from the project "COMFORT" (an Associate Team between INRIA project-team NeCS and the Berkeley University project PATH) and the project "Inria@SiliconValley".

\section{REFERENCES}

[1] A. Goel and P. Kumar, "Characterisation of Nanoparticle Emissions and Exposure at Traffic Intersections through Fast-Response Mobile and Sequential Measurements," Atmospheric Environment, vol. 107, pp. 374-390, 2015.

[2] T. Arsava, Y. Xie, N. Gartner, and J. Mwakalonge, "Arterial Traffic Signal Coordination Utilizing Vehicular Traffic Origin-Destination Information," in IEEE 17th International Conference on Intelligent Transportation Systems, 2014, pp. 2132-2137.
[3] Z. Tian and T. Urbanik, "System Partition Technique to Improve Signal Coordination and Traffic Progression," Journal of Transportation Engineering, vol. 133, no. 2, pp. 119-128, 2007.

[4] J. Morgan and J. Little, "Synchronizing Traffic Signals for Maximal Bandwidth," Operations Research, vol. 12, no. 6, pp. 896-912, 1964.

[5] J. Little, "The Synchronization of Traffic Signals by Mixed-Integer Linear Programming," Operations Research, vol. 14, no. 4, pp. 568594, 1966.

[6] J. Little, M. Kelson, and N. Gartner, "MAXBAND. A Versatile Program for Setting Signals on Arteries and Triangular Networks," Transportation Research Record: Journal of the Transportation Research Board, vol. 795, 1981.

[7] E. Chang, S. Cohen, C. Liu, N. Chaudhary, and C. Messer, "MAXBAND-86: Program for Optimizing Left-Turn Phase Sequence in Multiarterial Closed Networks," Transportation Research Record, no. 1181, pp. 61-67, 1988.

[8] N. Gartner, S. Assmann, F. Lasaga, and D. Hou, "A Multi-Band Approach to Arterial Signal Optimization Traffic," Transportation Research Part B: Methodological, vol. 25B, no. 1, pp. 55-74, 1991.

[9] C. Stamatiadis and N. Gartner, "MULTIBAND-96: A Program for Variable-Bandwidth Progression Optimization of Multiarterial Traffic Networks," Transportation Research Record, vol. 1554, pp. 9-17, 1996.

[10] N. Gartner and C. Stamatiadis, "Arterial-Based Control of Traffic Flow in Urban Grid Networks," Mathematical and Computer Modelling, vol. 35, no. 5-6, pp. 657-671, 2002.

[11] W. Xianyu, H. Peifeng, and Y. Zhenzhou, "Link-Based Signalized Arterial Progression Optimization with Practical Travel Speed," Journal of Applied Mathematics, vol. 2013, 2013.

[12] L. Lin, L. Tung, and H. Ku, "Synchronized Signal Control Model for Maximizing Progression along an Arterial," Journal of Transportation Engineering, vol. 136, no. 8, pp. 727-735, 2010.

[13] H. Tsay and L. Lin, "New Algorithm for Solving the Maximum Progression Bandwidth," Transportation Research Record, vol. 1194, pp. $15-30,1988$.

[14] G. Gomes, "Bandwidth Maximization Using Vehicle Arrival Functions," IEEE Transactions on Intelligent Transportation Systems, vol. 16, no. 4, pp. 1977-1988, 2015.

[15] J. Archer, N. Fotheringham, M. Symmons, and B. Corben, "The Impact of Lowering Speed Limits in Urban/Metropolitan Areas," Monash University Accident Research Center, Australia, Tech. Rep., 2008.

[16] G. De Nunzio, G. Gomes, C. Canudas de Wit, R. Horowitz, and P. Moulin, "Arterial Bandwidth Maximization via Signal Offsets and Variable Speed Limits Control," in IEEE 54th Annual Conference on Decision and Control, 2015, pp. 5142-5148.

[17] S. Boyd and L. Vandenberghe, Convex Optimization. Cambridge University Press, 2004.

[18] G. De Nunzio, C. Canudas de Wit, and P. Moulin, "Urban Traffic EcoDriving : A Macroscopic Steady-State Analysis," in European Control Conference, 2014, pp. 2581-2587.

[19] N. Petit and A. Sciarretta, "Optimal Drive of Electric Vehicles Using an Inversion-Based Trajectory Generation Approach," in IFAC 18th World Congress, 2011, pp. 14 519-14526.

[20] W. Dib, A. Chasse, A. Sciarretta, and P. Moulin, "Optimal Energy Management Compliant with Online Requirements for an Electric Vehicle in Eco-Driving Applications," in IFAC Workshop on Engine and Powertrain Control, Simulation and Modeling, 2012, pp. 334-340. 